\title{
Research on the Discrete-time Control System of Electromagnetic Speed Regulating Shearer
}

\author{
He Ming-xing ${ }^{*}$, Liu Jian-gong and Liu Yang
}

\begin{abstract}
School of Mechanical Electronic \& Information Engineering, China University of Mining and Technology (Beijing), Beijing 100083, China
\end{abstract}

\begin{abstract}
On the basis of review research on the control mechanism of the electromagnetic speed regulating shearer, a mathematical model with dual-loop control system of continuous-time system of electromagnetic speed regulating shearer was built. According to the control types and characteristics of each link in the continuous control system, the pulse transfer function was adopted in the discrete time domain to deduce the expression form of $\mathrm{Z}$ transformation parameters system of the discrete-time model. The stability of the system and operation characteristics of electromagnetic speed regulating shearer were analyzed on the $\mathrm{Z}$ plane; the response curves of discrete controller and frequency response curve of the system of the step signal were simulated in the zero-order holder. By combining the actual electromagnetic speed regulating shearer, the relevant application programs were written to respectively test the electromagnetic speed regulating shearer in the dual-closed loop control system under the conditions of no-load, free parking and the applied load so as to contrast the consistency of test data and simulation results.
\end{abstract}

Keywords: Discrete-time control system, electromagnetic speed regulating shearer, Z transformation.

In recent years, some coal mines have been mined for decades; thus coal reserves of the medium and thick coal seam with good geological conditions of reserves decrease rapidly. In order to guarantee the normal operation of the coal mines and effectively utilize mineral resources, the mining technology of thin coal seam has drawn great attention in the coal industry. Electromagnetic speed regulating shearer with the characteristics of simple structure, small volume, low failure rate, strong ability to adapt to the environment, has become a research hotspot for the thin coal seam shearer [1].

At present, there are three main forms of speed control technology in domestic thin seam coal shearers: switch reluctance, variable frequency and electromagnetic speed. The speed regulating technology of switch reluctance belongs to synchronous speed regulating technology. Its system is composed of a switch reluctance motor, a power converter, a controller and a position detector. Its regulating speed is of wide range and high efficiency. However, the synthesis torque is non constant torque, which has a great impact on the performance of low speed operation. [2-4] Variable frequency is a most widely-used asynchronous motor speed control technology. There are two kinds of speed control modes: the vector control and direct torque control. These control modes are of wide speed range, high precision and good dynamic response; however, they are needed to install the self-contained transformer in the application of the shearer, and its volume is limited. The electromagnetic speed adjusts the motor speed by changing slip ratio of the stator, and its system consists of an asynchronous motor and an electromagnetic clutch, which is of simple structure and smooth speed. But due to its soft natural mechanical properties, the controller will cause the instability of excitation operation current, leading to control failure. Therefore, it is needed to establish an appropriate mathematical model according to the specific operating conditions so as to realize the reliable control. In order to improve the work performance of electromagnetic speed regulating shearer, a model structure of the electromagnetic speed regulating shearer's control system based on the discrete time is proposed in this paper [5].

The control structure of electromagnetic speed regulating shearer is given in Ref 1, in which, the negative feedback of the motor speed is used to form a closed-loop control. The PID regulates the algorithm, which is applied to the current trigger circuit, adjusting excitation current, so as to change the speed of the motor [6]. The PID's control system consists of discrete device and logic circuit, which has realized the automatic control. The main drawback is that the control system is not designed under the condition of complete theoretical analysis, but obtained according to the experience value test. Furthermore, the PID parameters setting are not validated. Differential formulas are established for the excitation circuit of electromagnetic speed regulating motor $[2,7]$. According to the load characteristics of the shearer, the electromagnetic coupling load torque is studied. After the Laplace transformation, the transfer function of electromagnetic speed regulating shearer is derived and two closed-loop electromagnetic speed regulating shearers work model of the current and speed are designed. A PLC controller is used to conduct an experimental verification of the model that achieves better results. In the theoretical derivation, the first- 
order differential formula is used for the modeling. The formula is directly controlled by a digital system after linearization, and is not detailed in terms of data sampling and discretization analysis. In Ref 3, a double closed-loop electromagnetic speed regulating shearer model is adopted and a disturbance signal is introduced so that the whole system becomes more perfect. The simulink toolbox of MATLAB is used to conduct the simulation research on the whole system. Firstly, the dynamic performance and disturbance-resistant performance of the electromagnetic speed control system are simulated, and then the stability margin of the system is obtained. The simulation results show that the established model of electromagnetic speed regulating shearer has two closed loops with good stability and strong capacity of resisting disturbance; however, its system parameters and PID are almost the fixed values. And the result is generally applied to a narrow scope. In Refs 4 and 5, the electromagnetic speed controller is realized by using different control chips. The control strategy is based on the discrete system control, but the theoretical derivation is not carried on. In Ref6, a fuzzy control algorithm is proposed to realize speed regulation according to the traction properties and speed control characteristics of electric traction shearer [8-10]. The more traditional PID control method has a stronger adaptive capacity, but the setting of fuzzy rules needs a long time to accumulate. The computer system used in the industrial control is a typical discrete system, which has the advantages of high precision, high speed, flexible software and easy modification. In this paper, the Laplace transformation model of electromagnetic speed regulating shearer is improved. A digital model based on discrete time system is established, the $\mathrm{Z}$ transformation is used to deduce the pulse transfer function. The structure of double closed-loop feedback control system is established for discrete time, and its response performance and stability is analyzed so that it can be directly applied to the high-speed control of the embedded chip.

\section{ESTABLISHMENT OF SYSTEM CONTROL MOD- EL}

\subsection{Differential Formulas of the Motor Pulled by the Electromagnetic Speed Regulating Shearer}

Traction parts of the electromagnetic speed regulating shearer are one or two electromagnetic speed regulating motors. The motor is also called slip motor or slip clutch. The structure of the clutch is the electromagnetic clutch installed on the output shaft of the squirrel cage asynchronous motor. The excitation current of the clutch is controlled by an external device, and the size of excitation current is determined by the output speed of the clutch. Its half section is shown in Fig. (1). The prime mover drives the driving shaft to rotate and the stainless steel armature is installed on the driving shaft. The magnetic pole is installed on the driven shaft, which is composed of a core and the excitation winding. The load is driven by the driven shaft. When the direct current gets access to the coil of excitation winding, an alternating magnetic field is generated in turn in the circumference of the magnetic pole distribution. When the prime mover rotates, the magnetic line of armature cutting magnetic field generates eddy current in its interior, which interacts with theflux of rotating magnetic pole; thus the electromagnetic torque is generated in the tangent direction along the arma- ture, so as to drive the load to rotate along with the direction of the armature.

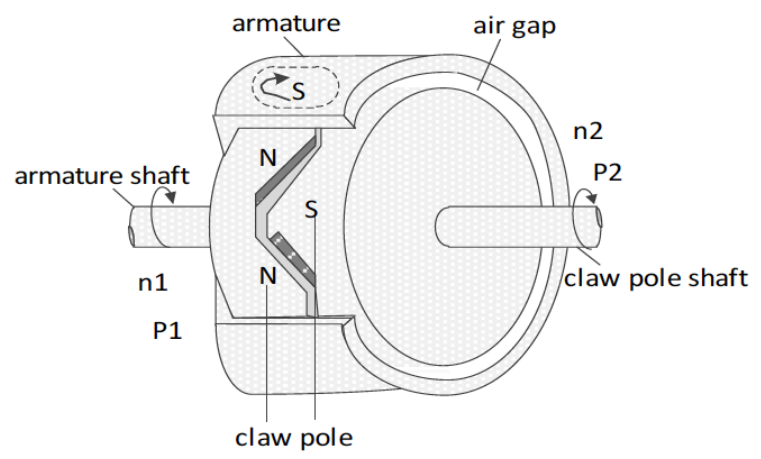

Fig. (1). Structure of magnetic clutch.

When the shearer works in the mine, its environment is very special and complex. The characteristics of load in the runtime cannot be easily described. Resistance received by the cutting unit of the shearer has a direct impact on the traction and the speed of the shearer. The inner coal seam has bedding and joints, and its stress intensity is different; therefore, the strength of coal walls is different as well [11]. Generally speaking, the strength of the lower coal wall is greater than that of the upper one. Tonsteins and nuclear pyrite are often contained in thick coal. When the shearer cuts these objects with high hardness, load resistance will radically change. In the control system, which is based on electromagnetic speed regulating shearer, the motor speed is regulated by means of the control method of variable torque based on the characteristics of the current load of the shearer. As a result, the motor speed can better adapt to the working characteristics of shearer and exert its best performance.

The magnetic field of electromagnetic regulating speed motor is established by the external excitation current and the output torque of the motor is generated by the eddy current in the armature mover [12].Its size is related to the induced electromotive force and its slippage. Temporarily, it is regarded as a first order linear constant system and the differential formulain the steady state can be expressed as follows:

$I_{B} R+L \frac{d I_{B}}{d t}+e_{d}=U_{d}$

In Formula1, $\mathrm{I}_{\mathrm{B}}$ is excitation current of the electromagnetic speed clutch in the excitation circuit. Ris the equivalent impedance of the magnetic pole in the excitation coil. L is inductance of excitation coil. $e_{d}$ is loss of electromotive force caused by the heat and magnetic flux leakage of magnetic pole winding eddy current. The generation of eddy current is constrained by the impact of winding parameters of electromagnetic speed motor, and is closely related to motor speed [13]. According to the general experience, the excitation constant is often set as $\mathrm{C}_{\mathrm{d}}$, therefore,

$\mathrm{e}_{\mathrm{d}}=\mathrm{C}_{\mathrm{d}} * \mathrm{n}$

It can be obtained after reorganization:

$\mathrm{I}_{\mathrm{B}} \mathrm{R}+\mathrm{L} \frac{\mathrm{dI}_{\mathrm{B}}}{\mathrm{dt}}+\mathrm{C}_{\mathrm{d}} \mathrm{n}=\mathrm{U}_{\mathrm{d}}$ 
To make the shearer operate in a stable state, the electric drive formula to be satisfies is as follows:

$T_{m}-T_{L}=\frac{G D^{2}}{375} \frac{d_{n}}{d_{t}}$

Wherein, $\mathrm{T}_{\mathrm{m}}, \mathrm{T}_{\mathrm{L}}$ are respectively electromagnetic torque and load torque. The size of $\mathrm{T}_{\mathrm{m}}$ is proportional to the velocity difference between armature and pole [14]. The constant of electromagnetic time is $T_{e}=\frac{L_{d}}{R}$. The constant of electromechanical time is $\tau_{\mathrm{m}}=\frac{\mathrm{GD}^{2} \mathrm{R}}{375 \mathrm{C}_{\mathrm{e}} \mathrm{C}_{\mathrm{t}}}$.

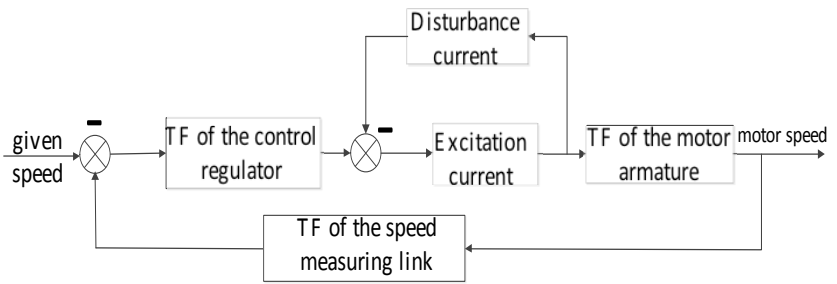

Fig. (2). The control structure diagram of electromagnetic speed regulating shearer.

In this system, the control signal generated by the velocity error signal adjusts the driving voltage of the electromagnetic clutch. The excitation current generated by the driving excitation coil establishes the armature magnetic field. During the operation of the motor, the power supply environment in the borehole is of poor quality. Fluctuations in the power system and other devices have an impact on the changes in the excitation current, resulting in an unstable motor field, thus it is very important to establish a current feedback control for the sake of the motor performance. The voltage of clutch is provided by the thyristor rectifier.

To make the system easy to analyze, during the derivation, the shearer is regarded as a traction model driven by as lip motor. A linear process is conducted for its high-order part. In practice, the system of the shearer works slowly. The power of the system is large and the velocity change shows a flat trend. The torques of the motors driven by the two slips are driven by a motor can be equivalent to a torque, which is of no impact on the control system [15].

\subsection{The Continuous Control Model for Electromagnetic Speed Regulating Shearer}

First of all, take a derivative transfer function of armature and rectifier of electromagnetic speed regulating shearer, and then use $\mathrm{Z}$ transform it to meet the need of computer digital discrete control. It can be obtained through Formula 3 and Formula 4.

$$
\left\{\begin{array}{c}
U_{d}-e_{d}=R\left(I_{b}+T_{e} \frac{d I_{B}}{d t}\right) \\
I_{m}-I_{L}=\frac{T_{m}}{R} \cdot \frac{d e_{d}}{d t}
\end{array}\right.
$$

Two sides of Formula 5are changed via the Laplace transform, it can be obtained as below:

$$
\left\{\begin{array}{c}
U_{d}(\mathrm{~s})-e_{d}(\mathrm{~s})=I_{a}(s) R\left(1+T_{e} s\right) \\
I_{m}(s)-I_{L}(s)=\frac{T_{m}}{R} e_{d} s
\end{array}\right.
$$

Formula 6 is sorted as transfer functions

$$
\left\{\begin{array}{c}
\frac{I_{a}(\mathrm{~s})}{U_{d}(s)-E(s)}=\frac{1 / R}{1+T_{e} S} \\
\frac{e_{d}(s)}{I_{m}(s)-I_{L}(s)}=\frac{R}{T_{m} s}
\end{array}\right.
$$

Armature structural chart composed of Formula 7 is shown in Fig. (3), and armature shows working condition of no-load rated voltage.

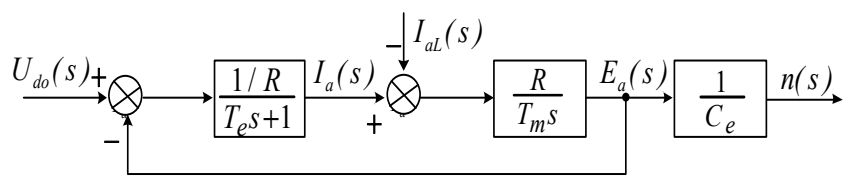

Fig. (3).The structure armature of electromagnetic speed regulating shearer.

In the figure, according to the equivalence principle, nodes of Ia (S) are moved forward and feedback nodes of $\mathrm{E}(\mathrm{s})$ arecombined. After these two terms are eliminated, the dynamic block diagram or the armature of electromagnetic speed regulating shearer can be simplified as shown in Fig. (4).

$$
\stackrel{U_{d}(s)}{\longrightarrow} \frac{1 / C_{d}}{T_{m} T_{e} s^{2}+T_{m} s+1} \stackrel{n(s)}{\longrightarrow}
$$

Fig. (4). The simplified structure armature of electromagnetic speed regulating shearer.

Its transfer function can be obtained as below:

$W_{d}=\frac{1 / C_{d}}{T_{m} T_{e} S^{2}+T_{m} S+1}$

Thyristor circuit includes trigger part and commutation control of rectifier, and is used to control voltage of excitation coil, so as to control magnetic flux of excitation. AC voltage triggers circuit breaking via pulse in order to control the opening of thyristor conduction angle and change the value of output voltage. Interval time of trigger pulse is $1 / \mathrm{mf}$ seconds. $\mathrm{M}$ and $\mathrm{f}$ refers to pulse frequency and power frequency respectively. Due to the conduction characteristic of thyristor, breaking time is uncontrolled, failure time of control hysteresisTs is 0 to $1 / \mathrm{mf}$ value, so amplified excitation part of thyristoris an amplification factor that belongs to pure lag amplification of Ks. Its function is expressed as below:

$W_{(\mathrm{s})}=\frac{C_{(s)}}{R_{(s)}}=K_{s} e^{-T_{S} s}$

The value of Ts is small, so higher-order term is simplified as $\mathrm{W}_{\mathrm{s}} \approx \frac{\mathrm{K}_{\mathrm{s}}}{1+\mathrm{T}_{\mathrm{s}} \mathrm{s}}$

For the system of electromagnetic speed regulating shearer, the single closed-loop of speed is used torealize floating operation of rotating speed of shearer. However, working conditions of coal minesarepoor. Current mutation occurs during the disturbance of the power grid, or the shearerenc ounters resistance when emergency stop and cut- 
ting occurs. The single closed-loop system is unable to completely control current and torque in the dynamic process according to the predetermined mathematical model, andthe speed regulating process is extended. Electromagnetic speed regulating shearer can directly change torque through exciting current, based on which, in the transient process of electromagnetic speed regulating shearer towards the steady state, running current is controlled within the maximum protective value [16]. When electromagnetic speed regulating shearer is stabilized, current is reduced and adjusted, so as to ensure torque equilibrium of shearer, and speed of shearer is controlled through speed regulation. Current and speed regulation adopts PI control. The general formula for transfer function is as follows:

$\left\{W_{A S R}(s)=K_{v}\left(\tau_{v} s+1\right) / \tau_{v} s\right.$

$\left\{W_{A C R}(s)=K_{i}\left(\tau_{i} s+1\right) / \tau_{i} s\right.$

Where $\mathrm{K}$ refers to proportionality coefficient of regulator, and ${ }^{\tau}$ refers to integral coefficient.

Formulas (5) to (9), armature model of electromagnetic speed regulating shearer, rectifier model and controller model constitute the dynamic structure diagram for electromagnetic speed regulating shearer, as shown in Fig. (5).

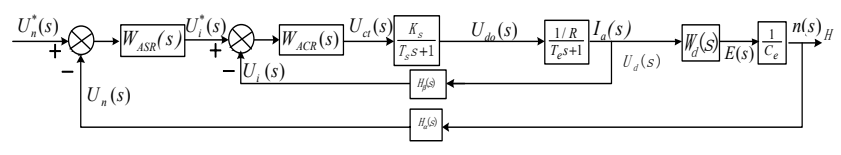

Fig. (5). The dynamic structure diagram of shearer.

\subsection{The Discrete Model for Pulse Transfer Function of Electromagnetic Speed Regulating Shearer}

Transfer function of continuous time system establishes a formula for input and output of continuous time signals. In a digital controller, output signal of continuous signals can be expressed by impulse sequence, as below.

$z[\mathrm{y}(\mathrm{t})]=\mathrm{Y}(z)=\sum_{k=0}^{\infty} y(\mathrm{kT}) z^{-k}$

Impulse sampling sequence of unit ideal sampler is defined as below:

$\delta_{\mathrm{T}}(\mathrm{t})=\sum_{\mathrm{k}=0}^{\infty} \delta(\mathrm{t}-\mathrm{kT})$

After outputting $y \quad(t)$, sampling switch $\mathrm{t}=\mathrm{kT}(\mathrm{k}=0,1,2 \ldots \ldots)$ is valued as below:

$\mathrm{y}^{*}(t)=\sum_{k=0}^{\infty} x(t) \delta(t-\mathrm{kT})=\sum_{k=0}^{\infty} x(k T) \delta(t-k T)$

Then

$\mathrm{y}(\mathrm{kT})=\sum_{h=0}^{k} g(k T-h T) x=\sum_{h=0}^{k} x(k T-h T) g(h T)$

And $\mathrm{Z}$ transform is as below:

$$
\begin{aligned}
\mathrm{Y}(z)=\sum_{\mathrm{k}=0}^{\infty} y(k T) & z^{-k}=\sum_{k=0}^{\infty} \sum_{h=0}^{\infty} g(k T-h T) x(h T) z^{-k} \\
& =\sum_{k=0}^{\infty} \sum_{h=0}^{\infty} g(m T) x(h T) z^{-(m+h)} \\
& =\sum_{m=0}^{\infty} g(m T) z^{-m} \sum_{h=0}^{\infty} x(h T) z^{-k} \\
& =G(z) X(z)
\end{aligned}
$$

Wherein, $G(z)$ is discrete pulse transfer function.

As for transfer function of armature link, when the pole is more two than null point at least, factorization can be conducted as below:

$$
\begin{aligned}
& W_{d}(S)=\frac{\frac{1}{C_{d}}}{T_{m} T_{e} s^{2}+T_{m} S+1}=\frac{r_{1}}{s-p_{1}}+\frac{r_{2}}{s-p_{2}}+k \\
& \text { So } \\
& \qquad \begin{array}{l}
W_{d}(\mathrm{kT})=\mathfrak{T}^{-1}\left[W_{d}(s)\right]=r_{1} e^{p_{1} k T}+r_{2} e^{p_{2} k T}+k \\
\qquad \mathrm{~W}_{d}(z)=\sum_{k=0}^{\infty} w(k T) z^{-k} \\
\quad=r_{1} \sum_{k=0}^{\infty}\left(e^{-p_{1} T} z\right)^{-k} \\
+r_{2} \sum_{k=0}^{\infty}\left(e^{-p_{2} T} z\right)^{-k}+k=\frac{r_{1}}{1-e^{-p_{1} T} z^{-1}} \\
\quad+\frac{r_{2}}{1-e^{-p_{2} T} z^{-1}}+k
\end{array}
\end{aligned}
$$

Transfer function of thyristor can be expressed as below:

$$
W_{s}(S)=\frac{K_{s}}{T_{S}} \frac{1}{\frac{1}{T_{s}}+S}
$$

$$
W_{S}(k T)=\frac{K_{S}}{T_{S}} e^{-\frac{1}{T_{S}} k T} \mathrm{k}=0,1,2 \cdots
$$

So

$$
\begin{aligned}
W_{S}(z)=\frac{K_{s}}{T_{S}} \sum_{0}^{\infty} e^{-\frac{1}{T_{S}} k T} z^{-k} & \\
& =\frac{K_{S}}{T_{S}} \sum_{0}^{\infty}\left(e^{-\frac{1}{T_{S}} T} z\right)^{-k}=\frac{K_{s}}{T_{S}} \frac{z}{z-e^{\frac{1}{T_{S}} T}}
\end{aligned}
$$

The digital current controller and speed digital controller of shearer are the closed-loop system. After the system is converted through sampling switch and $\mathrm{A} / \mathrm{D}$ conversion, the digital controller controls the operation of shearer through continuous control signals generated by zero-order holder.

Given that input error signal is $e(t)$, and output signal is $\mathrm{m}(\mathrm{t})$, the system of shearer is applicable to the position-type controller for trapezoidal summation, and pulse transfer function is expressed as below"

$$
\mathrm{m}(\mathrm{kT})=\mathrm{K}\left\{e(t)+\frac{T}{\tau}\left[\frac{\mathrm{e}(0)+\mathrm{e}(\mathrm{T})}{2}+\cdots+\frac{e((k-1) T)}{2}\right]\right\}
$$




$$
\begin{gathered}
=\mathrm{K}\left\{e(t)+\frac{T}{\tau} \sum_{h=1}^{k} \frac{\mathrm{e}((\mathrm{h}-1)) \mathrm{T}+\mathrm{e}(\mathrm{hT})}{2}\right\} \\
=\mathrm{K}\left\{e(t)+\frac{T}{\tau} \sum_{h=1}^{k} f(h T)\right\}
\end{gathered}
$$

$\mathrm{Z}$ transform is conducted, namely,

$\mathrm{M}(z)=\mathrm{K}\left(\mathrm{e}(z)+\frac{T}{\tau} \sum_{h=1}^{k} F(z)\right)$

Due to short sampling time of shearer, the formulas are as below:

$$
\begin{aligned}
Z(\mathrm{~F}(z))=\mathrm{f}(0)+\mathrm{f}(1) z^{-1}+\cdots=\frac{1}{1-z^{-1}} F(z) & \\
\mathrm{F}(z)=Z[\mathrm{f}(\mathrm{hT})] & =z\left[\frac{e((k-1) T+e(k T)}{2}\right] \\
& =\frac{1+z^{-1}}{2} E(z)
\end{aligned}
$$

For Speed control of outer loop, a sampling switch is set up in the rear end of comparing element, and its output is impulse sequences of error signal, PI degeneration control cannot be expressed by $\mathrm{Z}$ transform in parallel, so

$$
\frac{\mathrm{n}(z)}{u(z)}=\frac{W_{A S R}(z) C_{i}(z)}{1+W_{A S R} H_{\beta}(z)}
$$

It is respectively substituted into the expression of each factor and parameters concerning electric machine, so the formula for closed loop system function of electromagnetic speed regulating shearer is obtained as below:

$$
\mathrm{Y}(z)=\frac{\mathrm{b}_{0} z^{m}+\mathrm{b}_{1} z^{m-1}+\cdots+b_{m}}{\mathrm{a}_{0} z^{n}+\mathrm{a}_{1} z^{n}+\cdots+a_{n}}(\text { 其中 } \mathrm{n}>\mathrm{m})
$$

It is rewritten as a residue.

$$
\begin{gathered}
\mathrm{Y}(z)=\frac{(1.0341-0.4695 \mathrm{i}) z}{z-(0.9925+0.0109 \mathrm{i})}+\frac{(1.0341+0.4695 \mathrm{i}) z}{z-(0.9925-0.0109 \mathrm{i})} \\
+\frac{-1.6139 z}{z-0.5630}+\frac{-0.0123 z}{z-0.0049}
\end{gathered}
$$

Its zero-pole distribution is shown in Fig. (7).

It is substituted into the above formula, so it is obtained as below:

$\mathrm{M}(z)=\mathrm{K}\left(1+\frac{T}{2 \tau} \frac{1+z^{-1}}{1-z^{-1}}\right) E(z)$

Through Fig. (5) and Formula (10) to (17), the discrete control structure model for electromagnetic speed regulating shearer can be obtained as shown in Fig. (6).

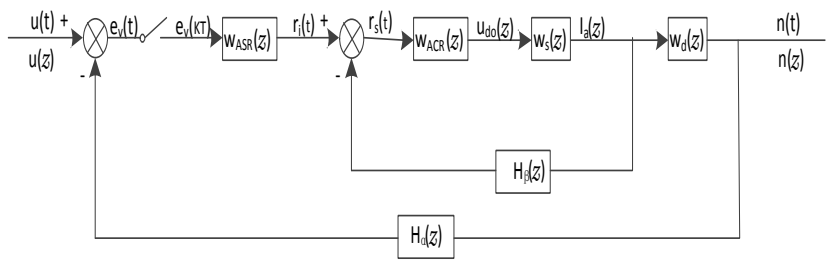

Fig. (6). The control diagram for discrete structure of electromagnetic speed regulating shearer.

\section{ANALYSIS OF SYSTEM SIMULATION}

A good exampleis "Shenma"brand electromagnetic speed regulating shearermade in Nanjing. The primary armature loop resistance is $\mathrm{R}=15 \Omega$, excitation current is $\mathrm{I}=5 \mathrm{~A}$, thyristor magnification factoris $\mathrm{Kr}=40$, stator-rotor mutual inductance is $2.5 \mathrm{~h}$, rotatory inertia is $0.12 \mathrm{~kg} / \mathrm{m} 2$, rotating speed feedback factor is $\alpha=0.05$, current feedback factor is $\beta=0.007$, and rated speed is $1360 \mathrm{r} / \mathrm{min}$.

\subsection{Zero-pole Analysis of Root Locus of Electromagnetic Speed Regulating Shearer}

Current controls inner loop in the system, so there is no sampling switch. Current controller and thyristor link are series equivalent, after PI feedback control, transfer link of current inner loopis equivalent as below:

$$
C_{\mathrm{i}}(z)=\frac{W_{A C R}(z) W_{s}(z) H_{\beta}(z)}{1+W_{A C R}(z) W_{S}(z) H_{\beta}(z)}
$$

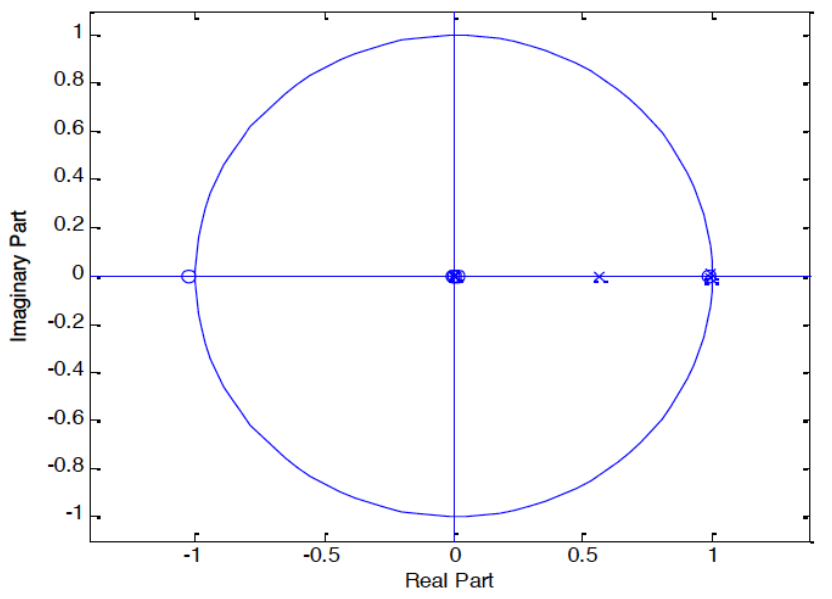

Fig. (7). The zero-pole point distribution diagram of discrete controller.

From the above diagram, it can be seen that all closedloop poles are within the unit circle of Z-plane, so the system is a stable regulating system. Pulse transfer function $\mathrm{Y}(\mathrm{z})$ of electromagnetic speed regulating shearer has two closedloop poles, which are located in the right half $\mathrm{Z}$ plane, on the real axis. Its response is a pulse convergent sequence. After analyzed from regulating characteristics of electromagnetic speed regulating shearer, it is found that speed regulation caused by external load needs a certain regulating time. In addition, current regulating speed caused by grid disturbance is fast, and its pole approaches the origin. Meanwhile, there is a pair of conjugate closed-loop plural pole, of which the modulus value is less than 1 , and its dynamic response is a pulse sequence of oscillatory convergence. Two poles are far away from the origin, so the convergence rate of system is slow. The shearer system needs a long time from regulating to its set point, and the system simulation results are consistent with the actual regulating process. Step signals are exerted on $\mathrm{Y}(\mathrm{z})$, under the action of the zero-order retainer, response is shown in Fig. (8). 


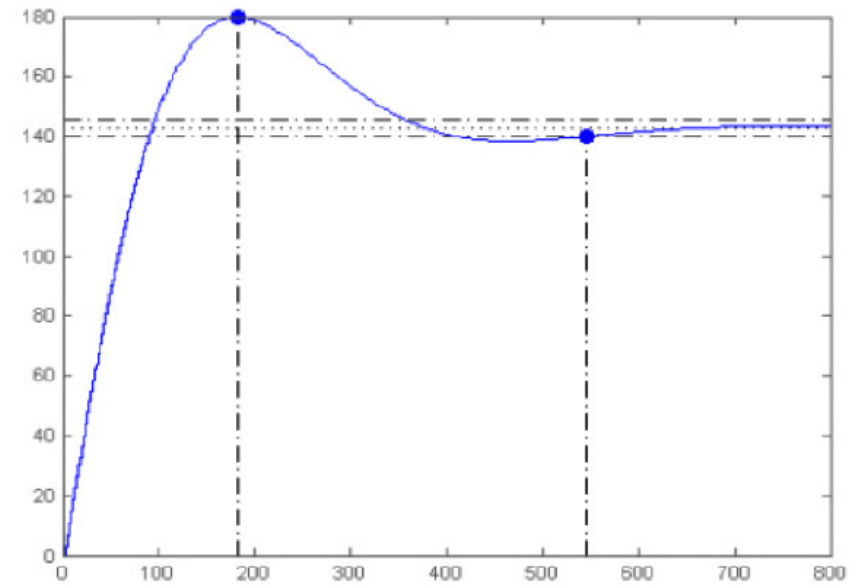

Fig. (8). The step response diagram of discrete controller.

The step response of electromagnetic speed regulating shearer is an under damped adjustment curve, and its overshoot is $16 \%$ of the system set point. The system has high oscillation intensity because electromagnetic clutch of shearer has the characteristics of soft mechanical adjustment and poor linear control. After a double closed-loop control algorithm is adopted, its convergence speeds up obviously, and attenuation rate is up to $85.6 \%$. After a considerable regulation, the system gradually enters into a steady regulating process. The steady state error of shearer is less than 3\% and the steady regulating time is long; thus the system keeps stable operation.

\subsection{Frequency Response of Shearer Discrete-time System}

In the continuous time system, given Laplace operator is $\mathrm{s}=\mathrm{jw}$, then frequency response of the system can be obtained, so as to verify frequency stability of the system and regulate it as shown in Table 1. In the frequency response test of the discrete time system, according to the relationship of complex variables $z$ and $s, z=e^{j w T}$. The left half $s$ plane is only mapped within the unit circle of $\mathrm{z}$ plane; therefore, through bilinear transformation $\mathrm{z}$ plane is converted to logarithmic coordinate calculation of $\mathrm{w}$ plane. Supposedlyz

$=\frac{1+(\mathrm{T} / 2) w}{1-(\mathrm{T} / 2) w}$

pulse transfer function of $\mathrm{z}$ plane is transformed into a rational function of $w$ plane, so as to adapt to the discrete extended system. The left half $\mathrm{w}$ plane corresponds to that of $\mathrm{s}$ plane. Bode diagram for the discrete time system of electromagnetic speed regulating shearer is shown in Fig. (9).

From the diagram, amplitude margin of the system is calculated at 0.9702 , its phase margin is- 0.9283 , its corner frequency is 9.7490 , and its ct-off frequency is 9.9108. In the amplitude-frequency curve, the asymptote attenuates monotonically and in its traversal, its phase margin is negative. The changing process is integration of the second order system and lag link response characteristics. The phasefrequency response curve is a horizontal asymptote at high frequencies, and control signals of electromagnetic speed regulating shearer have a small phase lag. Electromagnetic speed regulating shearer can control intermediate frequency and high frequency signals very fast and its frequency band is wide. Excitation signals of low frequency have a limited impact on the electromagnetic control process of electromagnetic speed regulating shearer. Nyquist diagram curve for electromagnetic speed regulating shearer is shown in Fig. (10):

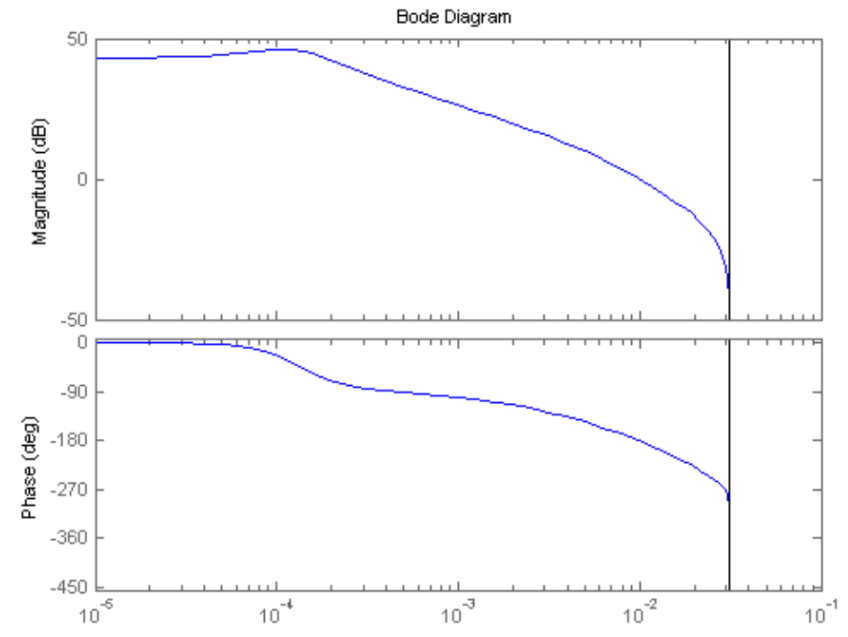

Fig. (9). The frequency response diagram of discrete controller.

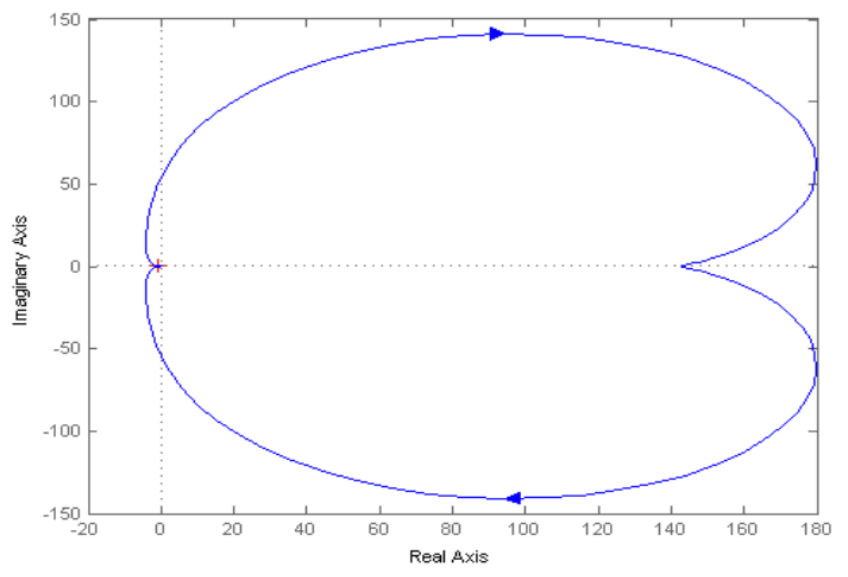

Fig. (10). The root-locus distribution diagram of discrete controller.

Open-loop transfer function of electromagnetic speed regulating shearerhas negative real rootin the splane only and no poleon the right half plane. In the diagram, Nyquistcurve is a clockwise encirclement and does not contain $(-1, j 0)$ internally. Based on Nyquistcriterion, it can be concluded that the electromagnetic speed regulating shearerhasa stable closed-loop system.

\section{SHEARER TEST AND ITSRESULT ANALYSIS}

According to the model and simulation data of discrete time of electromagnetic speed regulating shearer, the embedded Linux is used to establish the control system of circuit and software. The main control chip is AT91SAM9260, which has a running speed of $270 \mathrm{M}$. Its excitation circuit is composed of an intelligent thyristor module, and the maximum excitation current is $5 \mathrm{~A}$.AD collection and DA output program are written inC language with a sampling period of $5 \mathrm{~ms}$. The hardware structure of its electric control system is shown in Fig. (11). 
Table1. Test data for shearer unload

\begin{tabular}{|c|c|c|c|c|c|c|c|c|}
\hline Rotating speed & $\begin{array}{c}\text { The Set } \\
\text { point/r/min }\end{array}$ & $\begin{array}{c}\text { Static residual } \\
\text { error }\end{array}$ & $\begin{array}{c}\text { Stable re- } \\
\text { sponse time/S }\end{array}$ & $\begin{array}{c}\text { Prime mover } \\
\text { stop time/S }\end{array}$ & $\begin{array}{c}\text { Electromagnetic } \\
\text { clutch stop } \\
\text { time } / \mathrm{S}\end{array}$ & Overshoot/\% & $\begin{array}{c}\text { Excitation volt- } \\
\text { age } / \mathbf{V}\end{array}$ & $\begin{array}{l}\text { Excitation } \\
\text { current/A }\end{array}$ \\
\hline \multirow{3}{*}{ Ultra-low speed } & 50 & $-1 \sim+1$ & 61 & 45 & 65 & $2 \%$ & 1.6 & 0.097 \\
\hline & 100 & $-1 \sim+1$ & 110 & 37 & 67 & $1 \%$ & 2.0 & 0.128 \\
\hline & 200 & $-2 \sim+2$ & 48 & 60 & 10 & $1 \%$ & 2.6 & 0.175 \\
\hline \multirow{2}{*}{ Low speed } & 300 & $-2 \sim+3$ & 40 & 43 & 10 & $0.7 \%$ & 2.5 & 0.166 \\
\hline & 500 & $-3 \sim+5$ & 40 & 52 & 25 & $0.2 \%$ & 2.8 & 0.197 \\
\hline \multirow{2}{*}{ Medium speed } & 700 & $-9 \sim+8$ & 47 & 70 & 30 & $0.43 \%$ & 3.5 & 0.184 \\
\hline & 900 & $-12 \sim+7$ & 46 & 63 & 43 & $0.67 \%$ & 4.2 & 0.216 \\
\hline High speed & 1300 & $-13 \sim+13$ & 60 & 103 & 43 & $0.54 \%$ & 5 & 0.331 \\
\hline
\end{tabular}

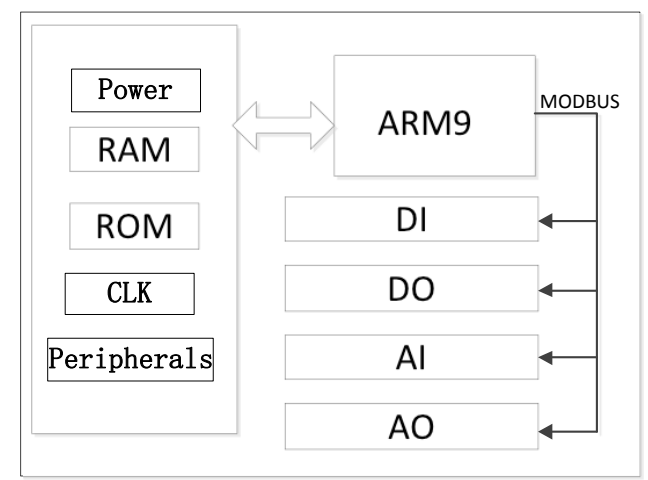

Fig. (11). The hardware structure of electromagnetic speed regulating shearer.

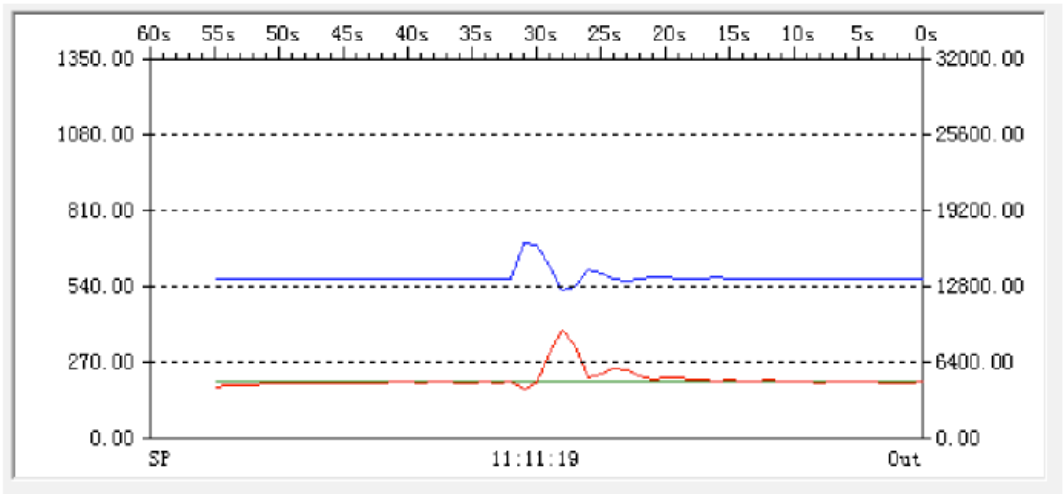

Fig. (12). The load characteristic of electromagnetic speed regulating shearer.

In no-load, electromagnetic clutch has less energy conversion, excitation circuit has less working current even when running at high speed after the shearer runs stably. Excitation current does not increase, so it can be seen that excitation circuit directly changes electromagnetic torque. Its control performance is stable, response speed is rapid, and overshoot is small.

After shearer runs stably, a dynamometer with an applied load of 95N.Mis used to test load characteristics of electromagnetic speed regulating shearer. Test results are shown in the figure. The speed of electromagnetic speed regulating shearer is set at 200 RPM. At the top is the output curve of regulating variable and in the center process is the feedback curve of process quantity motor speed. Its regulating process is basically consistent with the characteristics of simulation results, so as to verify the validity of the discrete time model. The results are shown in Fig. (12).

\section{CONCLUSION}

The control model for double-closed loop electromagnetic speed regulating shearer on the basis of the discrete time system is put forward in this paper and a type of discrete 
controller is designed to conduct the simulation test. Simulation and experimental results show the effectiveness of this method. At the same time, the shearer discrete control system based on the embedded computer is characterized by high speed, precision, large storage and strong software functions. According to the established digital model, it is easy to achieve complex functions. By improving software quality, the system's adaptability is also enhanced, and the operating indexes of the system are greatly increased.

\section{CONFLICT OF INTEREST}

The authors confirm that this article content has no conflict of interest.

\section{ACKNOWLEDGEMENTS}

Declared none.

\section{REFERENCES}

[1] S. Han, Optimal control and Application for Discrete-time Systems with Control delay, China Ocean University, 2012.

[2] X. Liu, and Z. Xie, "Design of electromagnetic speed regulation of shearers based on fuzzy control," Industrial Control Computer, vol. 05, pp. 55-58, 2009.

[3] J. Liu, and R. Wang, "Study of electromagnetically variable speed for excavators," Journal of China university of mining and Technology, vol. 01, pp. 107-110, 2005.

[4] W. Li, R. Wang, Y. He, and R. Fan, "Simulation of matlab of electromagnetic speed regulation control system in electrical shearer," Coal mine machinery, vol. 02, pp. 63-65, 2005.
[5] R. Ruan, M. Gong, and C. Yang, "Stability analysis of indirect adaptive regulation simple discrete-time system," Control Theory and Applications, vol. 04, pp. 666-672, 2005.

[6] B. Su, and X. Hua, "The Z-domain in Analysis of Discrete-time Systems Based on MATLAB," Journal of Tarim University, vol. 01, pp. 43-45, 2009.

[7] A. Tian, Study on PID Parameters Tuning of AC Drive System, The Northern Industrial University, 2011.

[8] L. Tong, "Design of double closed loop control system of ac-motor based on DSP," Modern Machinery, vol. 6, pp. 47-49, 2008.

[9] G. Wang, "A controller of electric-magnetic governing motor with PID regulating function," Guangdong Chemical Fiber, vol. 01, pp. 47-49, 1998.

[10] C. Xing, and J. Hang, "Based on DSP control system design of the electromagnetic variable-speed electric traction shearer," Electric Engineering, vol. 05, pp. 44-45, 2010.

[11] L. Yang, and A. Tian, "Discrete-time current regulator design for AC machine," Power Electronics, vol. 12, pp. 62-64, 2011.

[12] S. de. Yang, M. Gao, B. Zhou, and Y. Sun, "Closed-loop AC speed regulation system applying s-function," Coal Mine Machinery, vol. 10, pp. 41-43, 2011.

[13] J. Yan, and L. Ji, "Design of controller of electromagnetic speed regulating motor based on AVR sing-chip microcontroller," Industry and Mine Automation, vol. 11, pp. 60-63, 2009.

[14] S. Zhang, "Study on the Innovation of the fully mechanized coal shearer technology Research in China," Journal of China coal Society, vol. 11, pp. 1898-1902, 2010.

[15] L. Zhao, and M. Dong, "Load problems of working mechanism of the shearer in containing pyrites and thin coal seam," Journal of China coal Society, pp. 840-844, 2009.

[16] D. Zhang, T. Zhou, and Y. Liang, "Analysis of discrete-time system and realization based on MATLAB," Journal of Hunan institute of Science and Technology (natural science), vol. 01, pp. 3236, 2009.

Received: May 26, 2015
(C) Ming-xing et al.; Licensee Bentham Open.

This is an open access article licensed under the terms of the (https://creativecommons.org/licenses/by/4.0/legalcode), which permits unrestricted, noncommercial use, distribution and reproduction in any medium, provided the work is properly cited. 